\title{
Crowdfunding para la producción cultural basada en el procomún: el caso de Goteo (2011-2014)
}

\author{
Fátima SolERA NAVARro \\ Universidad de Málaga \\ mifasol.13@gmail.com \\ Florencio CABEllo FernándeZ-Delgado \\ Universidad de Málaga \\ fcabello@uma.es
}

Recibido: 1 de octubre de 2015

Aceptado: 22 de noviembre de 2015

\begin{abstract}
Resumen
La búsqueda de alternativas financieras tras el colapso bancario de 2008 atrae una creciente atención hacia el «crowdfunding». Este artículo parte de una revisión conceptual para luego centrarse en el «crowdfunding» de donaciones orientado a la producción cultural basada en el procomún. Para ello escogemos como caso de estudio la plataforma española Goteo, analizando los 158 proyectos lanzados en ella desde 2011 hasta mayo de 2014. Nuestra conclusión es que, si bien su recaudación es modesta y no muy diversificada, este modelo se consolida año a año, desafiando la visión neoliberal del «crowdfunding» como sustituto de la financiación pública a costa del agotamiento de las comunidades de apoyo.
\end{abstract}

Palabras clave: «Crowdfunding»; microfinanciación colectiva; procomún; cultura libre; industria cultural; Goteo.

\section{Crowdfunding for commons-based cultural production: the case of Goteo} (2011-2014)

\begin{abstract}
The search for financial alternatives after the 2008 banking collapse attracts a growing attention towards crowdfunding. This paper begins with a conceptual review and then focuses on donation-based crowdfunding linked to commons-based cultural production, selecting the Spanish platform Goteo as case study. We develop an exploratory analysis of the 158 cultural projects launched on Goteo from 2011 to May 2014. Even if the takings are modest and not very diversified, we conclude that this model grows stronger every year, thus challenging the neoliberal view of crowdfunding as a replacement for public funding at the expense of exhausting the supporting communities.
\end{abstract}

Keywords: Crowdfunding; collective microfunding; commons; free culture; cultural industry; Goteo.

\section{Referencia normalizada}

Solera Navarro, F. y Cabello Fernández-Delgado, F. (2015). Crowdfunding para la producción cultural basada en el procomún: el caso de Goteo (2011-2014). Historia y Comunicación Social. Vol 20, número 2, páginas 447-464. 
Sumario: 1. Introducción y estado de la cuestión. 1.1. Definición y categorías de «crowdfunding». 1.2. El «crowdfunding» en cifras: un sector en alza. 1.3. El «crowdfunding» en España. 1.4. «Crowdfunding» y producción cultural basada en el procomún: el caso de Goteo. 2. Metodología. 3. Resultados. 4. Discusión y Conclusiones 5. Agradecimientos. 6. Referencias bibliográficas.

\section{Introducción y estado de la cuestión}

El colapso bancario desencadenado en 2008 ha tenido un reflejo dramático en el bienestar social en España y ha secado el flujo de crédito a empresas y particulares. Según Coop57 (2014: 1), los créditos inferiores a 1 millón de euros a empresas españolas no financieras han caído un $66 \%$ desde 2008 , al tiempo que la factura del rescate bancario se eleva hasta los 220000 millones de euros entre ayudas directas e indirectas y la banca española acumula hasta 300000 millones en deuda pública de la que extrae cuantiosos dividendos.

Todo ello ha provocado la necesidad, y también la voluntad, de buscar alternativas para dotar de viabilidad financiera a múltiples entidades y proyectos desatendidos por la banca convencional. De este modo, asistimos al nacimiento y consolidación de iniciativas de banca ética como Fiare o Triodos Bank, de cooperativas de servicios financieros como Coop57 o, en lo que constituye el ámbito de interés del presente artículo, de diversas plataformas en línea de «crowdfunding».

Un episodio ilustrativo de estas transformaciones financieras en España lo encontramos en el sonado caso de las tarjetas opacas de CajaMadrid. El hecho de que este escándalo no se revelara por la denuncia de partidos políticos tradicionales sino gracias a la filtración de los correos de Blesa por la Comisión Anticorrupción del Partido X en colaboración con la iniciativa 15MpaRato (2014), y de que ambas organizaciones ciudadanas se sostengan económicamente gracias a campañas de «crowdfunding» (Partido X, 2014), es un ejemplo de cómo esta nueva forma de financiación empieza a afectar al sistema financiero tal y como lo conocemos.

\subsection{Definición y categorías de «crowdfunding»}

Son varias las traducciones que se han propuesto para el anglicismo «crowdfunding»: financiación en masa o masiva, micromecenazgo o, nuestra preferida, microfinanciación colectiva. En cualquier caso, estimamos que tal proliferación de traducciones refleja cierta confusión conceptual que deseamos despejar de inicio. Con tal fin aclararemos cómo lo definimos aquí y qué categorías distinguimos dentro de él, para a continuación explicitar a qué variante concreta de«crowdfunding» dedicaremos nuestro estudio.

Según Belleflamme, Lambert y Schwienbacher, el concepto de «crowdfunding» está enraízado en la noción más amplia de «crowdsourcing», por el cual una entidad externaliza en un colectivo de personas difusamente conectadas una tarea que previamente se realizaba en el seno de dicha entidad. A partir de esta raíz, Belleflamme, 
Lambert y Schwienbacher (2014: 586) especifican que el objetivo del «crowdfunding» es «recaudar dinero para inversiones, generalmente usando las redes sociales», si bien con la particularidad de que «en vez de solicitar el dinero a un pequeño grupo de sofisticados inversores multitud, el crowdfunding ayuda a las empresas a obtener dinero de audiencias amplias (la 'multitud'), en las que cada individuo proporciona una cantidad muy pequeña».

Por su parte, Sánchez González y Palomo Torres (2014: 102) ofrecen una visión que trasciende el ámbito estricto de la inversión financiera al considerarlo «un mecanismo de financiación de proyectos de diversa índole, mediante pequeñas aportaciones económicas de una gran cantidad de personas, y que, como apunta Burgueño (2011), combina la filosofía participativa de la web social con métodos creativos de búsqueda de financiación ciudadana».

Finalmente Rodríguez de las Heras (2015: 105-106) entiende el «crowdfunding» como una fórmula alternativa de financiación que surge de la convergencia de factores no solo económicos, sino también sociales (el auge de iniciativas de intervención colectiva y participación comunitaria) y tecnológicos (el entorno especialmente propicio para aquellas que se configura en internet). A partir de aquí, define el «crowdfunding» como «un amplio y variado fenómeno consistente en la creación de un entorno (electrónico) para la agrupación de un colectivo, la aportación de ideas, recursos y fondos y la interacción en red dirigidas a apoyar conjuntamente proyectos, esfuerzos e iniciativas de individuos, organizaciones o empresas» (ibid.: 107).

De estas definiciones pueden extraerse dos conclusiones fundamentales: la primera es el acento en la estrategia de financiación, en la medida en que es el alcance supuestamente amplio que alcanzan las convocatorias de «crowdfunding» mediante su proyección en internet lo que las distingue de formas clásicas de colecta o cuestación; la segunda es que bajo el paraguas conceptual del «crowdfunding» hallamos actividades muy dispares en su naturaleza e implicaciones. Ante ello resulta esencial deslindar conceptualmente las cuatro categorías de «crowdfunding» que distingue Massolution (2012: 12):

- «Crowdfunding» de inversiones: los inversores actúan como una suerte de (micro)capitalistas de riesgo, aportando una suma de dinero a cambio de participaciones en empresas o de porcentajes sobre sus beneficios futuros, con casos extremos como el de la plataforma Upstart, que permite convertirse en «accionista de un individuo» (Raim, 2014).

- «Crowdfunding» de préstamos: los inversores conceden préstamos a empresas o particulares (normalmente con la intermediación de una plataforma que analiza su viabilidad), y estos se comprometen a reintegrárselo con unos intereses estipulados con antelación, o incluso sin intereses, como en el caso de Kiva (Benkler, 2013: 232).

- «Crowdfunding» de recompensas: considerado una forma de preventa, se trata de una aportación dineraria orientada a la elaboración de artefactos tangibles 
y consumibles que, una vez producidos, se envían a los contribuyentes como contraprestación por su financiación.

- «Crowdfunding» de donaciones: los donantes efectúan aportaciones a proyectos sociales con los que sienten afinidad y no reciben contraprestación alguna, todo lo más el reconocimiento público o el envío de algún detalle simbólico. Esta es la categoría en la que se enmarca nuestra investigación, por lo que cuando usemos el término "crowdfunding» al exponer nuestros resultados y conclusiones estaremos aludiendo a esta variante específica.

Un buen ejemplo de la importancia de distinguir estas diversas categorías lo encontramos en la confusión suscitada por el Proyecto de Ley de Fomento de la Financiación Empresarial aprobado por el Gobierno de España en octubre de 2014. Así, pese a que la propuesta legal deja bien claro que su regulación va dirigida a las plataformas de internet que se dedican al «crowdfunding» de inversiones y de préstamos, no faltaron voces críticas que achacaban al Gobierno una voluntad de cerrar a proyectos sociales, culturales y políticos las vías de financiación mediante recompensas y, sobre todo, donaciones.

\subsection{El «crowdfunding» en cifras: un sector en alza}

Una primera referencia con datos correspondientes a 2012 es el «Crowdfunding Industry Report» de Massolution (2013), donde se expone que durante el año citado las plataformas de «crowdfunding», tomadas en su conjunto, facturaron 2700 millones de dólares en más de un millón de campañas exitosas. Ello suponía un incremento del $81 \%$ respecto a 2011 y llevaba a Massolution a predecir para 2013 un volumen de microfinanciación superior a 5000 millones de dólares. Con todo, estas halagüeñas perspectivas quedaron rebasadas ampliamente en el informe de Massolution de 2015, donde se cifra en 6100 millones de dólares el volumen de negocio finalmente alcanzado en el citado año 2013, volumen que asciende drásticamente hasta los 16200 millones en 2014 en virtud del «crecimiento exponencial» de las plataformas asiáticas (Crowdfundinsider, 2015). Otro dato importante del informe de Massolution (2013) era que de los 2700 millones facturados en 2012, un 59\% correspondía a plataformas norteamericanas y un 35\% a sus homólogas europeas, estimándose que en 2013 esta polarización geográfica no haría más que acentuarse. Pues bien, su último informe presenta un vuelco en esta clasificación, ya que si bien las plataformas norteamericanas retienen el liderazgo mundial en 2014, el citado auge de las plataformas asiáticas les han permitido rebasar a las europeas en el citado año, y las previsiones para 2015 indican que Asia ampliará en 4000 millones de dólares su diferencia de facturación respecto a Europa, cuya cuota de mercado se reducirá hasta un 18,8\% (Crowdfundinsider, 2015).

Si descomponemos los datos de Massolution (2013) en función de las categorías previamente expuestas, encontramos que en 2012 el 52\% del dinero se destinó a plataformas basadas en donaciones y recompensas, el $4 \%$ a plataformas de inversión y el $44 \%$ a plataformas de préstamo, lo que sitúa esta última variante como la de 
mayor crecimiento relativo (un $111 \%$ respecto a 2011). No obstante, los datos del último informe de Massolution dibujan un cambio de tendencia, siendo las plataformas vinculadas a la inversión (con fórmulas híbridas o basadas en royalties) las que experimentan un crecimiento más pronunciado frente a las de préstamos, donaciones y recompensas (Crowdfundinsider, 2015). Finalmente, y atendiendo a los sectores que más financiación recibieron, en 2012 las causas sociales mantenían el primer puesto (atrayendo casi el 30\% de las contribuciones), pero el sector empresarial y comercial recortaba distancias respecto a ellas (con un 16,9\%), seguido de los proyectos cinematográficos y escénicos $(11,9 \%)$, los musicales $(7,5 \%)$ y los vinculados al medioambiente y la energía (5,9\%) (Massolution, 2013). De nuevo los datos de 2014 (Crowdfundinsider, 2015) arrojan un panorama muy distinto, pues la categoría líder es ya indiscutiblemente la empresarial y comercial $(41,3 \%)$, seguida de las causas sociales $(18,9 \%)$, los proyectos cinematográficos y escénicos $(12,13 \%)$, el sector inmobiliario (que aparece como nueva categoría con una facturación de 6,25\%) y cierra la lista la categoría de iniciativas musicales $(4,54 \%)$.

Por su parte, Rusiñol (2014: 8) aporta dos datos interesantes para completar esta panorámica: por un lado, da cuenta de los récords de financiación alcanzados en EEUU por la plataforma líder «Kickstarter» (casi 1000 millones de euros desde su nacimiento en 2009) y por el proyecto líder en recaudación, el videojuego «Star Citizen» (37 millones de euros tras sucesivas campañas); por otro lado, recoge proyecciones de 2013 del Banco Mundial que prevén que las plataformas en línea de microfinanciación colectiva muevan un mínimo de 70000 millones de euros en 2025 (ibid: $6)$.

Con todo, el estudio más completo hasta la fecha es el informe comparativo que Wardrop, Zhang, Rau y Gray (2015) han elaborado para el Cambridge Centre for Alternative Finance con datos de 255 plataformas líderes de «crowdfunding» de 27 países europeos. Lo primero que destacan es el liderazgo apabullante del Reino Unido en este sector, tanto en volumen total (2337 millones de euros en 2014) como en volumen per cápita (36 euros por persona) y en ritmo de crecimiento respecto a $2013(168 \%)$. No obstante, los datos a escala europea revelan un florecimiento generalizado de la microfinanciación colectiva, con una facturación conjunta en 2014 de 2957 millones y un incremento del 144\% respecto a 2013 (respectivamente, 620 millones y $83 \%$ si se excluye al Reino Unido). Basándose en este ritmo de crecimiento, el estudio estima que el «crowdfunding» en Europa alcanzará en 2015 una facturación de más de 7000 millones de euros (más de 1300 millones si se excluye al Reino Unido).

Un último informe reseñable por poner su foco en el auge del «crowdfunding» es el «U.S. Economic Outlook» publicado por BBVA en julio de 2013. En él se analiza el nicho de mercado que atienden las plataformas de microfinanciación colectiva orientadas a inversiones y préstamos, reconociendo que «una característica importante de las innovaciones disruptivas es que comienzan sirviendo a la gama baja del mercado, es decir a segmentos que las grandes compañías pueden considerar no rentables» (BBVA, 2013: 31). Ello encaja con la teoría de la cola larga formulada por 
Chris Anderson (2004), que defiende que las redes de comunicación digitales han permitido una reducción de costes de transacción (más concretamente, de costes de almacenamiento y distribución) que posibilita que los modelos de negocio orientados a segmentos de mercado específico (a la cola de la distribución, donde mediante una suerte de personalización de masas se venden muy pocas unidades de muchísimos productos) sean tanto o más rentables que los tradicionales modelos de negocio masivos (orientados a la cabeza de la distribución, donde se venden muchísimas unidades de muy pocos productos).

Con todo, lo más significativo del informe es su advertencia ante la evolución futura que puede tomar el sector del «crowdfunding» más allá de la citada «cola larga»: «los crowdfundings de préstamos e inversiones son tecnologías disruptivas para la industria bancaria. [...] Quizá por primera vez en la historia, los negocios y los individuos tienen acceso a una fuente de capital sin precedentes originada en las pequeñas contribuciones de millones de individuos de todo el mundo» (BBVA, 2013: 30-33).

\subsection{El «crowdfunding» en España}

A la hora de traducir las perspectivas generales del sector a España, una primera referencia es el informe de 2014 de la Asociación Española de Crowdfunding (AEC). En él se estima que en 2013 la recaudación del sector a escala estatal fue de 19 millones de euros (AEC, 2014: 7) y se señala que la mayoría de plataformas $(65,2 \%)$ se dedica a recompensas y donaciones, frente a un $20,7 \%$ que lo hace a inversiones y un $13,8 \%$ a préstamos. En contraste, son estas dos últimas plataformas las que recaudan más dinero de media: frente al promedio de 4853 y 2025 euros que aportan respectivamente los inversores y prestamistas, las donaciones y precompras no llegan a los 40 euros de media por persona (ibid.: 6). Finalmente, resulta remarcable que de los 143 microfinanciadores que respaldan de media los proyectos exitosos (sin distinción de categorías) más de la mitad (74) hayan financiado más de un proyecto, lo que apunta un cierto componente de fidelización a la plataforma más allá de las aportaciones aisladas a iniciativas cercanas (ibid.: 9).

Por su parte, Wardrop, Zhang, Rau y Gray (2015: 33) ofrecen datos más actualizados y una perspectiva comparativa que permite ubicar a España en el mapa europeo del «crowdfunding». Para empezar, revisan muy al alza la estimación de la AEC sobre el volumen de recaudación en 2013 (cifrándolo en 29 millones de euros) y estiman el de 2014 en 62 millones, lo cual arroja una tasa de crecimiento anual del $114 \%$ (tasa que se eleva al 152\% si se considera desde 2012). En términos comparativos, tanto en 2014 como en el periodo 2012-2014 España ocupa la sexta posición en volumen total de financiación a escala europea, por detrás de Reino Unido, Francia, Alemania, Suecia y Holanda (ibid.: 15). No obstante, este mapa se trastoca al considerar el volumen per cápita, ya que España cae hasta la décima posición $(1,3$ euros por habitante) y Estonia se aúpa al segundo puesto con un gasto por habitante $(16,73$ euros) muy superior a los siguientes países del «ranking» (ibid.: 16). 
Por su parte, Rusiñol (2014: 8) completa esta radiografía del sector con el récord de recaudación alcanzado por una iniciativa española, actualmente ostentado por la empresa sevillana Gamezone Miniatures, que obtuvo 680000 euros en la plataforma Lánzanos para actualizar su juego de mesa «HeroQuest». La excepcionalidad de esta cifra queda patente si tenemos en cuenta que los proyectos de donación y recompensa exitosos (y el de «HeroQuest» pertenecía a esta última categoría) solicitan de media 3300 euros y tienden a fracasar si su petición sobrepasa los 5500 euros (AEC, 2014: $6)$.

En cuanto a las plataformas de «crowdfunding» en España, Martínez Gallardo y Alberich Pascual (2013: 90-91) mencionan como pionera a Partizipa, nacida en 2007 y encuadrada dentro de la categoría de microfinanciación colectiva orientada a inversiones. Tras ella, en 2010 nacen las dos plataformas líderes en España, Lánzanos y Verkami, que apuestan por las donaciones y precompras con una clara vocación de promoción cultural. Un año después surgen las dos últimas plataformas que destacan los autores mencionados, Kifund y Goteo. A esta última dedicaremos los siguientes apartados, por lo que nos limitamos a destacar a Kifund como una iniciativa basada en recompensas que «merece ser reconocida como la primera plataforma de crowdfunding en España y en el conjunto de países de habla hispana especializada únicamente en proyectos audiovisuales» (ibid.: 91). Para culminar esta panorámica del sector en España, cabe reseñar el estudio de «casos de éxito» divididos por categorías de «crowdfunding» que realiza Martínez Albaladejo (2014: 28-40) y el detallado análisis de Roig y Soriano (2015) sobre el «crowdfunding» de préstamos para PyMEs españolas.

\section{4. «Crowdfunding» y producción cultural basada en el procomún: el caso de Goteo}

Como se ha visto, las principales plataformas de «crowdfunding» de España están aún fuertemente vinculadas con la financiación de proyectos culturales. En este sentido, cabe destacar de entrada el temprano análisis de Hernanz (2012) sobre la viabilidad del «crowdfunding» cultural en España a través de la plataforma Verkami. De él se desprende que el $73 \%$ de los proyectos nacionales impulsados por Verkami concluyó satisfactoriamente su financiación, si bien esta cifra asciende al 75,3\% si sumamos los proyectos procedentes de Europa y América. El desglose por sectores muestra que los proyectos editoriales son los que tienen una mayor tasa de éxito $(81,1 \%)$, por encima de los musicales $(79,1 \%)$, pese a ser estos los que más recurren a la financiación. Por su parte, las iniciativas sociales alcanzan una tasa de éxito próxima al $75 \%$. En el otro extremo, los proyectos que peor resultado obtienen son los festivales, pues el 84,2\% fracasan en su intento de conseguir financiación colectiva en Verkami. En cuanto a las cantidades solicitadas, Hernanz concluye que aquellas iniciativas con presupuestos inferiores registraron una mayor tasa de fracaso, mientras que a medida que aumenta la cifra requerida aumenta considerablemente la tasa de éxito, destacando sobremanera la franja de los $10000 €$, a partir de la cual los proyectos alcanzan una tasa de éxito del $100 \%$. 
En este contexto, nuestra elección de Goteo como caso de estudio se fundamenta en su carácter pionero, no ya a escala nacional sino mundial, como iniciativa de «crowdfunding» de donaciones volcada a un tipo particular de producción cultural, aquella basada en el procomún: «Goteo es una plataforma de crowdfunding o financiación colectiva (aportaciones monetarias) y colaboración distribuida (servicios, infraestructuras, microtareas) para proyectos que además de ofrecer recompensas individuales generen retornos colectivos. Esto es, que fomenten el procomún, el código abierto y/o el conocimiento libre» (Goteo, 2014b). Para captar mejor la singularidad de esta plataforma, estimamos ineludible definir qué es el «procomún», en tanto concepto nuclear de su funcionamiento.

Según Yochai Benkler (2015: 98-99), el procomún es «una específica forma institucional de estructurar el derecho de acceso, uso y control de los recursos» que se opone a la propiedad privada en tanto que, en lugar de asignar a alguien el control exclusivo de los mismos, establece que «cualquier miembro de un grupo (más o menos definido) de personas puede usar o disponer de los recursos regidos por el procomún, de acuerdo con unas normas que pueden ir desde el "todo vale" a reglas formales escrupulosamente articuladas que se aplican de modo efectivo». En este sentido, puntualiza que la expresión «basada en el procomún» alude a que «la característica de las empresas cooperativas que describo [es que] los insumos y productos del proceso son compartidos, de manera libre o condicional, en un marco institucional que los hace igualmente disponibles para que todos los usen a discreción» (ibid.: 100).

En la misma línea, Antonio Lafuente (2007: 16) apostilla: «Lo que aquí nos interesa es subrayar cómo hemos ido apartándonos de la noción de propiedad para adentrarnos en la de comunidad. Y es que es imposible evitar lo que es obvio: el procomún, los bienes comunes -los «commons», en inglés- sostienen y son sostenidos por colectivos humanos. [...] De la ética de los valores hemos de transitar a la de las capacidades si queremos entender cómo es la dinámica de producción del procomún, pues un bien común no es más que una estrategia exitosa de construcción de capacidades para un colectivo humano».

A la luz de lo expuesto, se entiende que el compromiso fundamental con que la fundación no lucrativa Fuentes Abiertas gestiona Goteo desde su lanzamiento en 2011 sea que los proyectos culturales devuelvan a las comunidades que les respaldan financieramente una serie de beneficios que trasciendan las contraprestaciones individuales. Un ejemplo paradigmático de ello es el requisito de difundir con licencias libres el código fuente informático, las piezas artísticas, las producciones culturales, la documentación técnica o los datos en bruto generados por la iniciativa financiada. En este sentido, Goteo apuesta por aportar proximidad y especificidad a través de un sistema distribuido con comunidades locales y/o temáticas. Otra peculiaridad de Goteo es que contempla dos fases de recaudación: una primera de cuarenta días para alcanzar los fondos mínimos con que poner en marcha el proyecto; y, en caso de éxito, una segunda de igual duración para alcanzar fondos óptimos con que culminarlo. Por último, es reseñable que su plataforma tecnológica (cuyo código fuente es 
a su vez libre) puede funcionar y ser administrada de manera autónoma por categorías temáticas o geográficas, y mediante una comunidad de revisores de proyectos que los asesoran y validan.

\section{Metodología}

La presente investigación tiene como objetivo calibrar el grado de viabilidad financiera de los proyectos culturales basados en el procomún que emplean Goteo como plataforma de «crowdfunding», así como identificar posibles factores que faciliten dicha viabilidad con vistas a su consideración en futuras campañas. Más concretamente, nos proponemos averiguar los siguientes indicadores:

- Qué tasa de éxito alcanza este tipo de proyectos, tanto en su conjunto como anualmente y según su procedencia geográfica.

- Qué cantidad media (tanto mínima como óptima) solicitan los proyectos, qué cifra obtienen en promedio los proyectos exitosos y qué porcentaje representa esta sobre el mínimo requerido.

- Qué promedio de personas contribuyen económicamente a los proyectos exitosos y qué cifra media aportan.

- Qué cantidad mínima y máxima se establece para las donaciones individuales.

- Cuántas colaboraciones no monetarias se requieren de media, cuántas personas se ofrecen a cubrirlas y qué porcentaje de respuesta se alcanza.

- Qué categorías de recompensas individuales y retornos colectivos existen.

- Cuántas publicaciones se realizan durante la campaña y cuántos comentarios generan.

Para alcanzar estos objetivos hemos realizado un análisis exploratorio sobre una base de datos de elaboración propia a partir de la información financiera disponible en Goteo. El procesamiento de dicha base de datos nos ha permitido obtener los datos tanto cuantitativos (básicamente dinero y número de colaboraciones no monetarias) como cualitativos (categorías de proyectos, tipos de recompensas y retornos colectivos, zonas geográficas...) con los que en una segunda indagamos los indicadores previamente citados.

Nuestra base de datos abarca un total de 158 proyectos auspiciados por Goteo entre el 13 de diciembre de 2011 y el 11 de mayo de 2014 (fechas correspondientes al plazo límite de recaudación). Todos los proyectos estudiados comparten la categoría cultural, es decir, se encuadran dentro de la plataforma como proyectos culturales. Debido a la gran diversidad de temáticas que este concepto puede comprender y a que la propia plataforma no aplica más diferencias dentro del sector, decidimos ceñirnos a esta categorización global, si bien de los 158 proyectos 132 se encuadran además como mínimo en una segunda categoría (social, comunicativo, tecnológico...). 


\section{Resultados}

El primer resultado destacado de este estudio alude al éxito de las campañas de microfinanciación colectiva en Goteo, lo cual nos lleva a distinguir tres estados de los proyectos culturales estudiados:

- 52 proyectos «archivados» (que no consiguieron el mínimo necesario para financiar su proyecto).

- 102 proyectos «cofinanciados» (que obtuvieron al menos el mínimo necesario para financiar su proyecto)

- 4 proyectos catalogados como «misión cumplida» (que consiguieron al menos el mínimo y además han publicado ya en Goteo el retorno colectivo prometido).

Para la mejor visualización de los resultados asignaremos un color a cada categoría: el morado a los proyectos «archivados», el azul a los «cofinanciados» y el verde a los de «misión cumplida». Por último, el gris se empleará para aludir al total de iniciativas.

Figura 1. Porcentaje de proyectos «archivados», «cofinanciados» y «misión cumplida»

\section{0 \\ 00000 \\ $67 \%$ de los proyectos}

$33 \%$ archivados

$64,5 \%$ cofinanciados

$2,5 \%$ misión cumplida

Fuente: Elaboración propia ${ }^{1}$

Atendiendo a las categorías en las que se encuadran los proyectos objeto de estudio, encontramos que las tres principales son «cultural» (que engloba a 27 proyectos), «social, educativo, y cultural» (18) y «social, comunicativo, educativo y cultural» (13). En cuanto al dinero mínimo y óptimo solicitado para financiar las iniciativas, la Figura 2 muestra las cifras medias globales y disgregadas por proyectos «cofinanciados» $\mathrm{y}$ «archivados», quedando patente que los primeros demandan un promedio notablemente superior a los segundos. 
Figura 2. Promedios mínimo y óptimo

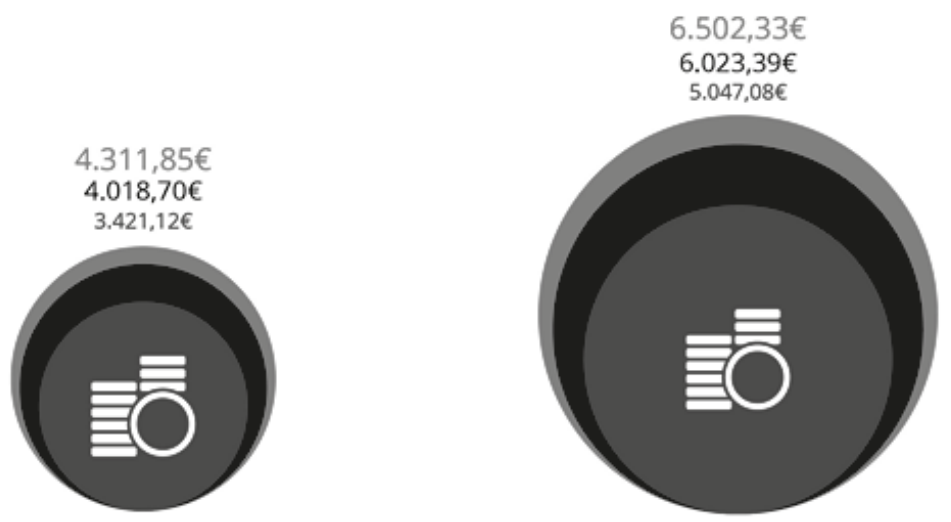

Promedio mínimo necesario

Promedio óptimo

Fuente: Elaboración propia

Con respecto a la recaudación obtenida por los proyectos «cofinanciados», asciende a un total de 564126 euros, con una media de 5321,94 euros por iniciativa, la cual supone un $123,42 \%$ respecto del promedio mínimo solicitado que vimos en la Figura 2. En cuanto al número de «cofinanciadores», encontramos un total de 11252 y una media de 106,15 por proyecto exitoso, de lo cual se desprende una media de 50,13 euros aportados por persona. El mínimo de «cofinanciadores» que encontramos es 5 (que reunieron 4415 euros para el proyecto «La Comuna de París de Louise Michel») y el máximo es 427 (que aportaron 20734 euros al último disco de D'Callaos).

Si atendemos a la evolución histórica desde diciembre de 2011 a mayo de 2014, identificamos claramentre una tendencia al alza, no solo en el número de proyectos culturales que se lanzan en Goteo, sino sobre todo en aquellos que obtienen como poco el mínimo solicitado. Esto se traduce en una tasa de éxito que asciende del 56,89\% en 2012 al 68\% en 2013 hasta alcanzar el 87,5\% en 2014.

$\mathrm{Al}$ estudiar las cantidades mínimas y máximas que los creadores de proyectos establecen para las contribuciones individuales, observamos una gran homogeneidad, sin que existan apenas diferencias al respecto entre los proyectos «cofinanciados» y «archivados». 
Figura 3. Cifra media de aportaciones mínimas y máximas solicitadas

Aportación mínima

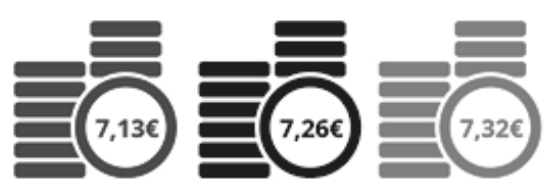

Aportación máxima

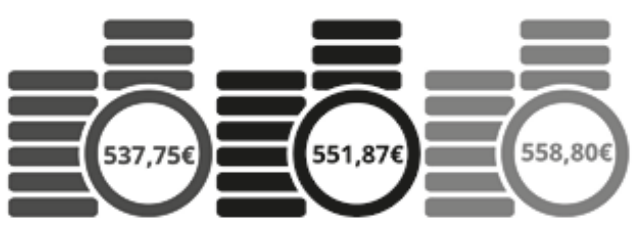

Fuente: Elaboración propia

En cuanto a los tipos de recompensa individual que los creadores ofrecen a sus «cofinanciadores», tampoco hallamos diferencias entre proyectos exitosos o no, pues todos ofrecen, en primer lugar, «reconocimiento, productos y servicios» y, en segundo lugar, «archivos digitales, reconocimiento, productos y servicios». Acaso el tipo más original de recompensa la ofreciera la Fundación Robo en su exitosa campaña de 2012 para la edición de un disco y DVD por su primer aniversario, pues quienes aportaran la máxima cantidad de 900 euros (y tres personas lo hicieron) adquirían el derecho a un concierto privado.

Si nos fijamos en los tipos de retorno colectivo que ofrece cada proyecto, encontramos la misma similitud sea cual sea el estado final del mismo, lo cual nos lleva a descartar este factor como decisivo del éxito o fracaso del «crowdfunding» orientado al procomún. Así, los dos retornos colectivos predominantes en Goteo son, en este orden, los «archivos digitales» y el conjunto de «archivos digitales y servicios».

Otro dato interesante es la Comunidad Autónoma de la que proceden los proyectos culturales estudiados. De este modo, observamos que la Comunidad de Madrid concentra la mayoría de proyectos presentados (34), seguida muy de cerca por Cataluña (32) y a mayor distancia por Andalucía (17) y País Vasco (16). Sin embargo, las tornas cambian si atendemos al número de proyectos «cofinanciados», pues Cataluña toma la delantera con 23 (lo que supone una tasa de éxito del 71,85\%), seguida de Madrid con 20 (58,82\%), País Vasco con 12 (75\%) y Andalucía con 10 (58,82\%). Es importante añadir que un total de 9 proyectos se ubican en más de una Comunidad Autónoma, o en España en su conjunto.

Aunque Goteo está radicado en España, con el tiempo se ha abierto a iniciativas procedentes de otros países. Así, encontramos campañas de financiación de proyectos culturales procedentes de México (2), Colombia (2), EEUU (1), Nicaragua (1), Gambia (1), Alemania (1), Francia (1) y Andorra (1), de las cuales se cuentan seis exitosas y cuatro «archivadas». 
La política de transparencia que Goteo aplica a su funcionamiento interno también la exige a los proyectos que alberga, los cuales deben desglosar adónde se destinará el dinero que recaudan. Este desglose se divide en tres categorías de recursos (infraestructura, materiales y tareas) para las cuales se establecen a su vez sendos presupuestos mínimos y óptimos. En la Figura 4 presentamos el promedio de dichas cifras consideradas de forma global y diferenciada entre proyectos «archivados» y «cofinanciados». Podemos observar que las cifras mínimas y óptimas más modestas corresponden siempre a la categoría de infraestructura, y ello independientemente del éxito o no de la campaña. Por contra, las tareas aglutinan el grueso de las demandas monetarias, si bien la demanda mínima de los proyectos exitosos está bastante por debajo de la demanda mínima global (1878,22 euros) mientras que su demanda óptima supera al promedio óptimo global (2991,48 euros).

Figura 4. Desglose de presupuestos mínimos y máximos por categorías de recursos

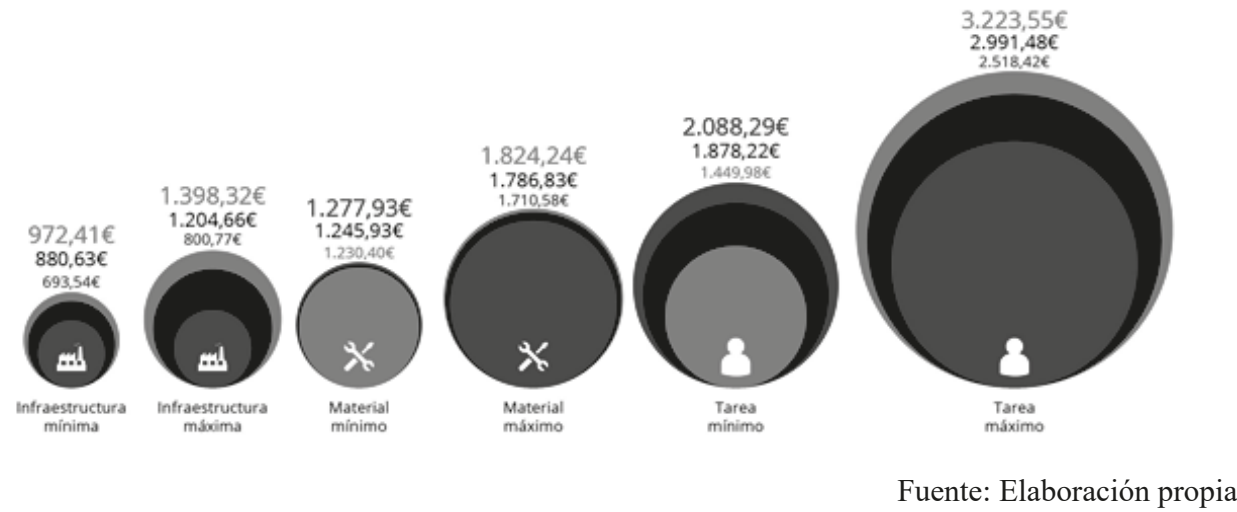

Si desplazamos el foco a las contribuciones no monetarias y estudiamos cuántas solicita el proyecto inicialmente y cuántas personas se ofrecen a cubrirlas, lo primero destacable es el elevado porcentaje de respuesta, que llega al 344,92\% de media en el caso de los proyectos exitosos. En total, los proyectos estudiados atrajeron contribuciones no monetarias de 1013 personas, dato muy importante si consideramos que Goteo permite que los proyectos «archivados» también se beneficien de ellas (por más que la mayoría se concentre en iniciativas «cofinanciadas»). 
Figura 5. Colaboraciones solicitadas, colaboradores obtenidos y porcentaje de respuesta por tipo de proyecto.

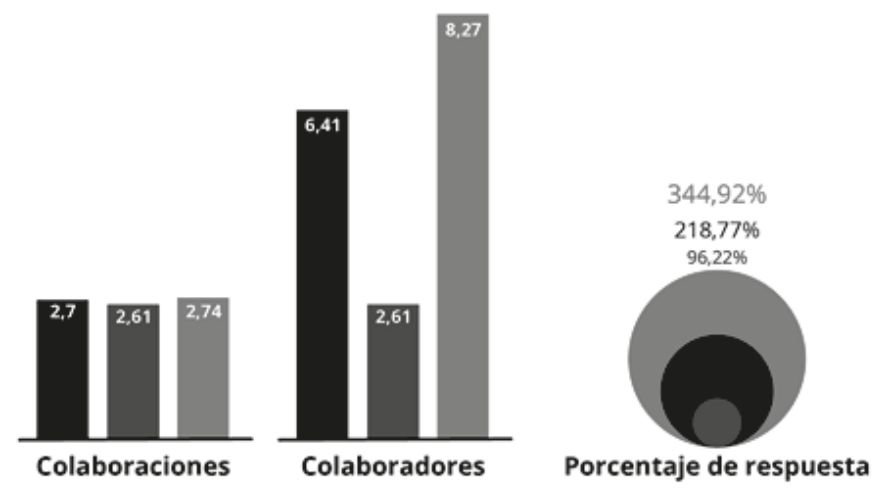

Fuente: Elaboración propia

Para finalizar, nos referimos a dos aspectos importantes para valorar el esfuerzo comunicativo que realizan los proyectos con objeto de mantener la atención durante los días que dura el «crowdfunding»: por un lado, estudiamos la media de actualizaciones publicadas en el blog que Goteo habilita para ello, y por otro cuántos comentarios generan dichas entradas. Como se aprecia en la Figura 6, la media de publicaciones de novedades de los proyectos «archivados» no llega ni a la mitad de la de los «cofinanciados», diferencia que se agranda en el caso de los comentarios, si bien con el matiz de que ni siquiera los proyectos exitosos llegan a un comentario por publicación.

Figura 6. Novedades y comentarios.

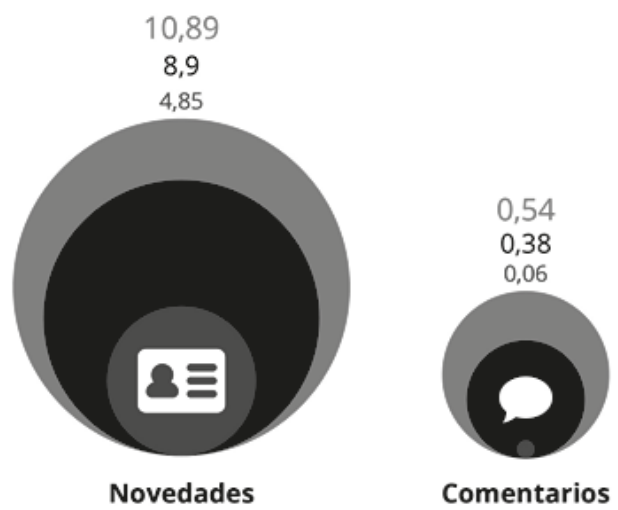

Fuente: Elaboración propia 


\section{Discusión y conclusiones}

Lo primero que deseamos subrayar aquí es la importancia de precisar la definición del término «crowdfunding» $\mathrm{y}$, más concretamente, de adoptar y extender la distinción entre las cuatro categorías citadas al principio: «crowdfunding» de inversiones, de préstamos, de recompensas y de donaciones. No se trata solo de un afán académico de rigor conceptual que evite confusiones entre ámbitos tan dispares como, por ejemplo, las finanzas y la filantropía (confusiones que además permean la sociedad a medida que se extiende su uso). Se trata también de una conditio sine qua non para poder desarrollar estudios comparativos (por categorías, sectores, volumen de recaudación, países, periodos temporales) a partir de la creciente disponibilidad de datos cuantitativos sobre lo que Wardrop, Zhang, Rau y Gray (2015) denominan «finanzas alternativas».

Centrándonos en los datos cuantitativos expuestos arriba sobre los proyectos culturales promovidos por Goteo, empezamos señalando lo relevante de la cifra de recaudación de dichos proyectos (564 126 euros) respecto del total de recaudación (2 millones de euros) que la plataforma obtuvo hasta mediados de año (Goteo, 2014a). En cuanto a la tasa de éxito de las campañas de financiación de estos proyectos, si bien es cierto que el $67 \%$ alcanzado por Goteo entre 2011 y 2014 no alcanza la media de $75,3 \%$ que vimos en Verkami (Hernanz, 2012), no lo es menos que este porcentaje aumenta año a año, alcanzando el $87,5 \%$ de enero a mayo de 2014. Estimamos además que dicha tendencia al alza se verá reforzada por la decisión de Goteo (2015) de reducir su comisión del $8 \%$ al 4\%, lo cual la sitúa en la franja más baja del sector.

Otra conclusión significativa es que no por requerir una cantidad mínima más elevada disminuyen las probabilidades de éxito de los proyectos culturales presentados en Goteo, como prueba nuestro hallazgo de que el promedio mínimo requerido por los proyectos «cofinanciados» $(4311,85$ euros) supera con creces el de los «archivados» $(3421,12)$ e incluso la propia media global de 3300 euros que AEC (2014: 7) estima para los «crowdfundings» de recompensa y donación en España en 2013. Teniendo en cuenta que Hernanz (2012) llega a la misma conclusión respecto de los proyectos culturales financiados en Verkami, podemos encontrarnos ante una singularidad del «crowdfunding» cultural (al menos en España) que resultaría interesante corroborar en futuras investigaciones.

Igualmente reseñable resulta que la media de 50,13 euros que los «cofinanciadores» aportan a los proyectos culturales estudiados no solo rebase la media nacional de 40 euros por persona estimada por la AEC (2014: 6) sino también el promedio de 47 euros que Goteo (2014a) obtiene globalmente. Con todo, no podemos ignorar el límite de «escalabilidad» (X.net, 2012: 13) que estos datos evidencian, en la medida en que las cifras aún exiguas recaudadas en España solo permiten impulsar proyectos culturales de escala o recorrido modestos.

Más allá de aspectos cuantitativos, deseamos subrayar que la relevancia que otorgamos al «crowdfunding» orientado al procomún no radica en su particularidad o 
exotismo, como si nos interesara atender a un nicho específico del sector de la microfinanciación colectiva. Antes bien, para nosotros este enfoque, y su pionera encarnación práctica en Goteo, representa el paradigma del «crowdfunding» por cómo supera las dos principales críticas que se vienen formulando al micromecenazgo convencional.

La primera crítica realiza una carga de profundidad a la supuesta redistribución de riqueza que facilitaría virtualmente el «crowdfunding» para alertar sobre el «agotamiento de las comunidades» (ibid.: 16): «El éxito de la financiación de los diferentes proyectos está directamente vinculado con la capacidad que tengan sus instigadores de movilizar a sus comunidades más cercanas y a los grupos sociales directamente beneficiados por los mismos. [...] La incapacidad de aumentar o regenerar estas comunidades supone un reto colectivo que aún no se ha asumido». En sus formulaciones más aceradas, esta crítica llega a equiparar el «crowdfunding» con un «esquema piramidal» (Macphee, 2012) que «monetiza las relaciones sociales» (Ridgway, 2013: 159). A la luz de estas críticas, el énfasis de Goteo en los retornos colectivos emerge como una apuesta crucial por la sostenibilidad social del «crowdfunding»: «Debería asumirse que un tipo de financiación como el crowdfunding que hace expresamente un llamamiento a "la comunidad" para realizarse, debería plantear como inherente a su propia naturaleza la idea de producir siempre un retorno a "la comunidad"» (X.net, 2012: 18).

La segunda crítica inscribe el auge del «crowdfunding» en el actual contexto de recortes a la financiación pública de la cultura y alerta del peligro de considerarlo un reemplazo de esta que apuntale en el ámbito cultural la lógica neoliberal. Sin llegar a idealizar unas políticas culturales que también dejan bastante que desear (Ridgway, 2013: 157; Zemos98.org, 2015), destacamos que Goteo contribuye a redefinir críticamente el papel de la inversión pública en línea con la apuesta mencionada arriba: «Las administraciones públicas deberían enfatizar y apoyar aquellas plataformas que propician un retorno social de los productos generados [y] fomentan la transparencia y el desglose económico de los proyectos que acogen» (X.net, 2012: 18). Nos referimos concretamente a las convocatorias que Goteo bautiza como «capital riego», en las que las administraciones públicas (aunque también pueden ser privadas) realizan una primera selección de proyectos que luego lanzarán un «crowdfunding» donde cada aportación particular será complementada por una contribución institucional equivalente «para lograr un efecto multiplicador y así fomentar la inversión co-responsable en proyectos que cuenten con el apoyo de la sociedad civil» (Goteo, 2014c).

En última instancia, en el ámbito de la microfinanciación colectiva resuena una tensión fundamental en los actuales debates sobre la llamada «economía de compartición» entre la lógica neoliberal del «empresario de sí mismo, que es su propio capital, su propio productor, la fuente de sus ingresos» (Foucault, 2007: 265) y la solidaridad social implícita en la «producción entre iguales basada en el procomún» (Benkler, 2015: 98-100). 


\section{Agradecimientos}

Agradecemos al equipo de Goteo, y especialmente a María García Perulero, su apoyo y asesoramiento.

\section{Referencias bibliográficas}

AEC (ASOCIACIÓN ESPAÑOLA DE CROWDFUNDING) (2014). Informe de la encuesta de la Asociación Española de Crowdfunding sobre las plataformas españolas de crowdfunding. (http://goo.gl/clBXKU).

ANDERSON, C. (2004). The Long Tail. Wired, 12 (10). (http://goo.gl/ZtxXjU).

BBVA RESEARCH (2013). US Economic Outlook. (http://goo.gl/VO306d).

BELLEFLAMME, P., LAMBERT, T. \& SCHWIENBACHER, A. (2014). Crowdfunding: Tapping the right crowd. Journal of Business Venturing, 29, 5, 585-609. (doi:10.1016/j.jbusvent.2013.07.003).

BENKLER, Y. (2015). La riqueza de las redes. Barcelona: Icaria.

(2013). Practical Anarchism: Peer Mutualism, Market Power, and the Fallible

State. Politics \& Society, 41, 2, 213-251. (doi:10.1177/0032329213483108)

FOUCAULT, M. (2007). El nacimiento de la biopolítica. Buenos Aires: Fondo de Cultura Económica.

LAFUENTE, A. (2007). Los cuatro entornos del procomún. Archipiélago, 77-78, 15-22.

MACPHEE, J. (2012, noviembre). Who's the Shop Steward on Your Kickstarter? The Baffler, 21. (http://goo.gl/3144je).

MARTÍNEZ ALBALADEJO, M. (2014). Crowdfunding: una alternativa de financiación online. TFG en Administración y Dirección de Empresas (Universidad Politécnica de Cartagena). (http://goo.gl/n8Gw9I).

MARTÍNEZ GALLARDO, F. D. \& ALBERICH PASCUAL, J. (2013) Plataformas y proyectos de Crowdsourcing y crowdfunding cinematográfico en España (20072011). Historia y Comunicación Social, 18, 85-95. (doi:10.5209/rev_HICS.2013. v18.43950).

MASSOLUTION (2012). Crowdfunding Industry Report: Market Trends, Composition and Crowdfunding Platforms. (http://goo.gl/vD0VG).

(2013). 2013CF. The Crowdfunding Industry Report. (http://goo.gl/2vm1f3).

RAIM, L. (2014, julio). Conviértase en accionista... de un individuo. Le Monde Diplomatique, 20.

RIDGWAY, R. (2013). Crowdfunding: Monetizing the Crowd?. En BAZZICHELLI, T. \& COX, G. (Eds.). Disrupting Business: Art and Activism in Times of Financial Crisis (pp-153-174). Nueva York: Autonomedia. (http://goo.gl/fkuXg0).

RODRÍGUEZ DE LAS HERAS, T. (2015). El crowdfunding: una forma de financiación colectiva, colaborativa y participativa de proyectos. Pensar en Derecho, 3, 101-123. 
ROIG, J. y SORIANO, J.M. (2015). Crowdfunding de préstamos para PyMEs en España: Un análisis empírico. Estudios de Economía Aplicada, 33, 301-318. (http://goo.gl/SvSZpb).

RUSINOL, P. (2014, septiembre). La eclosión del 'crowdfunding' cambia todas las reglas. Alternativas Económicas, 17, 6-9.

SÁNCHEZ GONZÁLEZ, M. \& PALOMO TORRES, M.B. (2014). Conocimiento y valoración del «crowdfunding» en Comunicación: La visión de profesionales y futuros periodistas. Comunicar, 43 (22), 101-110. (doi:10.3916/C43-2014-10).

WARDROP, R., ZHANG, B., RAU, R. \& GRAY, M. (2015). Moving Mainstream. The European Alternative Finance Benchmarking Report. (http://goo.gl/lmBWvV).

X.NET (2012). Experiencias de Crowdfunding en el Estado español y Cataluña. (http://goo.gl/f3YBkZ).

6.1 Referencias web

COOP57 (2014, 25 de abril). Boletín informativo. (http://goo.gl/eOtlb9).

CROWDFUNDINSIDER (2015, 31 de marzo). Massolution Posts Research Findings: Crowdfunding Market Grows 167\% in 2014, Crowdfunding Platforms Raise \$16.2 Billion. (http://goo.g1/LG797A)

GOTEO (2015, 25 de marzo) Solo 4\% de comisión solidaria. (http://goo.gl/Jat8PR). (2014a, 27 de julio). Pidiéndole más al crowdfunding: resultados abiertos que generan impacto social. (http://goo.gl/iL0wbU).

(2014b). FAQ: ¿Qué es Goteo? (http://goo.gl/LfnpmN). (2014c). Capital riego. (http://goo.gl/0REuNm).

HERNANZ, M. (2012, 9 de octubre). ¿Funciona el crowdfunding cultural en España? RTVE.ES. (http://goo.gl/N7ChZ).

PARTIDO X (2014, 27 de junio). Las cuentas claras y los tiempos también. (http:// goo.gl/eYSXsr).

ZEMOS98.org (2015, 11 de marzo). El Festival Zemos98 se ahogó. (http://goo.gl/ JKE3qS).

15MPARATO (2014, 4 de octubre). Cómo salieron a la luz las tarjetas negras de Caja Madrid: \#LaCiudadaníaLoHizo. (http://goo.gl/OifuJL).

\section{Notas}

1 Figuras basadas en iconos de Simpleicon (Figuras 2 y 3), Elegant Themes (Figura 4), Freepik (Figuras 4 y 6) y Yannick (Figura 6). (http://goo.gl/8gWOIS) 